\title{
Residual dipolar couplings by off-magic-angle spinning in solid-state nuclear magnetic resonance spectroscopy
}

\author{
Giuseppe Pileio, Ying Guo, Tran N. Pham, John M. Griffin, \\ Malcolm H. Levitt, Steven P. Brown
}

\section{(S1) Full reference abbreviated in main text}

Ref. 8:

Lai, W. C.; McLean, N.; Gansmüller, A.; Verhoeven, M. A.; Antonioli, G. C.; Carravetta, M.; Duma, L.; Bovee-Geurts, P. H. M.; Johannessen, O. G.; de Groot, H. J. M.; Lugtenburg, J.; Emsley, L.; Brown, S. P.; Brown, R. C. D.; DeGrip, W. J.; Levitt, M. H. J. Am. Chem. Soc. 2006, 128, 3878.

\section{(S2) Experimental Details}

Experiments were performed on a Varian/Chemagnetics Infinity+ spectrometer operating at a ${ }^{1} \mathrm{H}$ frequency of $300 \mathrm{MHz}$ using a $4 \mathrm{~mm}$ Bruker double resonance probe. The MAS frequency was set to $13.130 \mathrm{kHz}$. The ${ }^{1} \mathrm{H}$ and ${ }^{13} \mathrm{C} 90^{\circ}$ pulse lengths were 2.5 and $4.0 \mu \mathrm{s}$, respectively. The recycle delay was $3 \mathrm{~s} .{ }^{13} \mathrm{C}$ transverse magnetisation was created by ramped cross polarisation ${ }^{1,2}$ from ${ }^{1} \mathrm{H}$ using a contact time of $1 \mathrm{~ms} .{ }^{1} \mathrm{H}$ TPPM decoupling $^{3}$ was employed, using a rf field corresponding to a nutation frequency of $\omega_{1} / 2 \pi=100 \mathrm{kHz}$.

For spin-echo (CP $\left.-\tau / 2-\pi-\tau / 2-t_{2}\right)$ experiments, (see Fig. S1) the spin-echo intervals $\tau / 2$ were set to an integer number of rotor periods. An 8-step phase cycle was employed to select $\Delta p= \pm 1$ (2 steps) and \pm 2 (4 steps) on the ${ }^{1} \mathrm{H} \pi / 2$ and ${ }^{13} \mathrm{C} \pi$ pulses, respectively ( $\Delta p$ refers to the change in coherence order, $p$ ). For each $\tau$ value, 16 transients were co-added. The TPPM decoupling parameters giving the longest spin-echo

dephasing time, $T_{2}$, for $\mathrm{U}^{13} \mathrm{C}$ glycine were determined by direct spectral optimisation ${ }^{4,5}$ for a spin-echo duration, $\tau=30 \mathrm{~ms}$. The optimized TPPM pulse length and phase were 
$4.76 \mu$ s and $14.6^{\circ}$, respectively. In spin-echo experiments, a short acquisition time of 5 ms was used; line broadening was applied before Fourier transformation to avoid truncation artefacts.

U-13 C glycine was obtained from Cambridge Isotope Laboratories, MA, USA and used without further purification - the observed ${ }^{13} \mathrm{C}$ chemical shifts are indicative of the $\alpha$ polymorph. $^{6}$

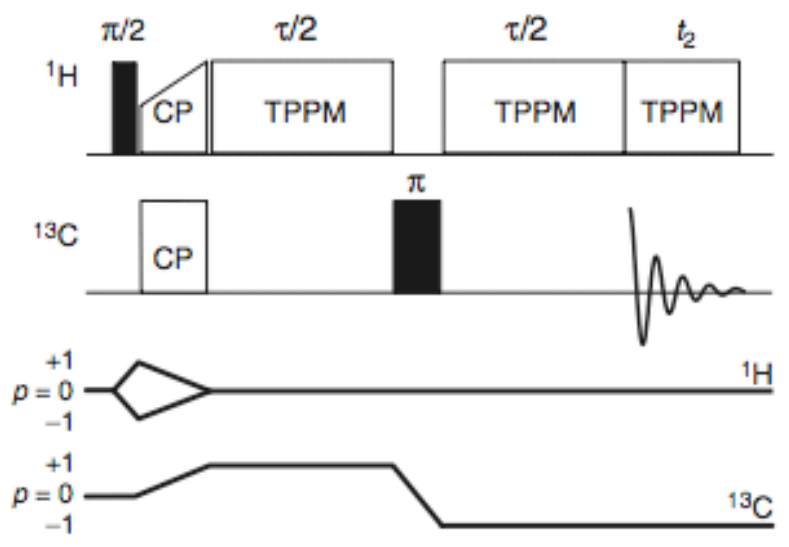

Figure S1 Pulse sequence and coherence transfer pathway diagram for the spin-echo experiment.

\section{(S3) Theory of off-MAS echo modulation curves}

In ref. ${ }^{7}$, a theoretical treatment was given for rotor-synchronized spin echoes, in the case of J-coupled pairs of spins-1/2 in exact magic-angle spinning experiments. This theory is readily adapted to the case of off-magic-angle spinning.

The spin Hamiltonian for two spins $S_{j}$ and $S_{k}$ is written as follows:

$$
H_{j k}=\omega_{j} S_{j z}+\omega_{k} S_{k z}+\omega_{A} 2 S_{j z} S_{k z}+\omega_{B} \frac{1}{2}\left(S_{j}^{+} S_{k}^{-}+S_{j}^{-} S_{k}^{+}\right)
$$

where the two coupling terms depend on the direct and indirect dipole-dipole coupling as follows:

$$
\begin{aligned}
& \omega_{A}=\pi J+d \\
& \omega_{B}=2 \pi J-d
\end{aligned}
$$


where $d$ is the secular component of the through-space dipole-dipole coupling. In a rotating sample, the chemical shift frequencies $\omega_{j}$ and $\omega_{k}$, and the secular dipole-dipole coupling $d$, are time-dependent.

The time-dependent terms are expressed as a Fourier series as follows:

$$
\omega_{Q}=\sum_{m=-2}^{2} \omega_{Q}^{(m)} \exp \left\{i m \omega_{r} t\right\}
$$

where $\omega_{r}$ is the spinning frequency and $Q$ refers to any of $A, B, j$ or $k$. In the exact magicangle-spinning case, the zero-order Fourier components are given by

$$
\left.\begin{array}{c}
\omega_{j}^{(0)}=\omega_{j}^{i s o} \\
\omega_{k}^{(0)}=\omega_{k}^{i s o} \\
\omega_{A}^{(0)}=\pi J \\
\omega_{B}^{(0)}=2 \pi J
\end{array}\right\}
$$

However, this is not true for off-magic-angle spinning. The most important term is the zero-order Fourier component of $\omega_{A}$, which is given in general by

$$
\omega_{A}^{(0)}=\pi J+b d_{00}^{2}\left(\beta_{P R}\right) d_{00}^{2}\left(\beta_{R L}\right)
$$

Here $b$ is the dipole-dipole coupling constant, $d_{00}^{2}\left(\beta_{P R}\right)=\frac{1}{2}\left(3 \cos ^{2} \beta_{P R}-1\right)$ is an element of the second-rank reduced Wigner matrix, $\beta_{P R}$ is the angle between the internuclear vector and the rotor axis, and $\beta_{R L}$ is the angle between the rotor axis and the static magnetic field. The angle $\beta_{P R}$ is a random variable for a powder sample, while $\beta_{R L}$ is equal to $\arctan \sqrt{2}$ for exact magic-angle spinning.

If the spinning angle is given by $\beta_{R L}=\arctan \sqrt{2}+\Delta$, where the angle misset $\Delta$ is small, the coupling Fourier component may be approximated by

$$
\omega_{A}^{(0)} \cong \pi J+b \Delta 2^{1 / 2} d_{00}^{2}\left(\beta_{P R}\right)
$$

As described in ref. ${ }^{7}$, the echo modulation signal contains one component which is strongly orientation-dependent and which damps quickly in a powder sample, and one component which is only weakly orientation-dependent and which survives powder 
averaging almost unscathed. The following phenomenological form is therefore appropriate for the powder-average signal:

$$
s(\tau)=p \exp \left\{-\tau / T_{2}^{0}\right\}+(1-p) s_{\text {mod }}(\tau, \Delta) \exp \left\{-\tau / T_{2}^{J}\right\}
$$

where $T_{2}^{0}$ and $T_{2}^{J}$ are phenomenological dephasing time constants. The time constant $T_{2}^{J}$ corresponds to the homogeneous decay of the $J$-modulated component, while the time constant $T_{2}^{0}$ takes into account an inhomogeneous spread of modulation components with different modulation frequencies (see section 4 of Ref. ${ }^{7}$ ).

The modulated component, averaged over a powder, is given by

$$
s_{\text {mod }}(\tau, \Delta)=\left\langle\cos \left(\omega_{A}^{(0)}\left(\beta_{P R}, \Delta\right) \tau\right)\right\rangle
$$

The angular brackets denote powder averaging:

$$
S_{\text {mod }}(\tau, \Delta) \cong \frac{1}{2} \int_{0}^{\pi} \cos \left(\pi J \tau+b \Delta 2^{1 / 2} d_{00}^{2}\left(\beta_{P R}\right) \tau\right) \sin \beta_{P R} \mathrm{~d} \beta_{P R}
$$

The integral is taken over a single angle and has the same form as is encountered in $\gamma$ encoded recoupling sequences. ${ }^{8}$ The following analytical solution may be derived using the techniques described in ref. $^{8}$ :

$$
s_{\text {mod }}(\tau, \Delta)=x^{-1}\left(F_{c}(x) \cos \theta+F_{s}(x) \sin \theta\right)
$$

where

$$
\begin{aligned}
& x=\left(\frac{6 b \tau}{\pi \sqrt{2}}\right)^{1 / 2} \\
& \theta=\left(\pi J+2^{-1 / 2} b \Delta\right) \tau
\end{aligned}
$$

and $F_{c}(x), F_{s}(x)$ are the cosine and sine Fresnel integrals:

$$
\begin{aligned}
& F_{c}(x)=\int_{0}^{x} \cos \left(\frac{\pi y^{2}}{2}\right) \mathrm{d} y \\
& F_{s}(x)=\int_{0}^{x} \sin \left(\frac{\pi y^{2}}{2}\right) \mathrm{d} y
\end{aligned}
$$

For the case of exact magic-angle spinning $(\Delta=0)$, Eq. (E10) reduces to the form 
$S_{\text {mod }}(\tau, 0)=\cos \pi J \tau$

(E13)

as used in ref. ${ }^{7}$.

\section{(S4) Full Fitting Details}

Table S1 presents fits of the spin-echo data in Fig. 1 and Fig. 2 to Eq. (E7) where $s_{\text {mod }}(\tau, \Delta)$ is given by Eqs. (E10-E12). The spin-echo data sets, $s(\tau)$ were obtained, after Fourier transformation, by integrating over the ranges 165 to $191 \mathrm{ppm}\left(\mathrm{CO}_{2}^{-}\right)$for Fig. 1 and 35 to 52 ppm $\left(\mathrm{CH}_{2}\right)$ for Fig. 2 .

\begin{tabular}{|c|c|c|c|c|c|c|}
\hline & $\mathbf{J} / \mathbf{H z}$ & $\Delta / \circ$ & $\mathbf{T}_{2}{ }^{0} / \mathrm{ms}$ & $\mathbf{T}_{2}{ }^{\mathrm{J}} / \mathbf{m s}$ & $\mathbf{p}$ & RMSD $* * / H z$ \\
\hline \multicolumn{7}{|c|}{ Fig. 1} \\
\hline $\mathbf{a}$ & $56.0 \pm 0.2$ & $0.0^{*}$ & $36 \pm 12$ & $46.1 \pm 0.2$ & $0.11 \pm 0.02$ & 0.02 \\
\hline $\mathbf{b}$ & $56.0^{*}$ & $-0.27 \pm 0.01$ & $36 \pm 6$ & $119 \pm 7$ & $0.15 \pm 0.01$ & 0.04 \\
\hline \multicolumn{7}{|c|}{ Fig. 2} \\
\hline $\mathbf{a}$ & $56.6^{*}$ & $-0.49 \pm 0.01$ & $1.7 \pm 0.2$ & $45 \pm 5$ & $0.17 \pm 0.04$ & 0.02 \\
\hline $\mathbf{b}$ & $56.6^{*}$ & $-0.26 \pm 0.01$ & $26 \pm 8$ & $36 \pm 2$ & $0.04 \pm 0.01$ & 0.04 \\
\hline c & $56.6 \pm 0.2$ & $0.0^{*}$ & $26 \pm 9$ & $26 \pm 2$ & $0.03 \pm 0.02$ & 0.02 \\
\hline d & $56.6^{*}$ & $0.26 \pm 0.01$ & $4.6 \pm 0.4$ & $42 \pm 5$ & $0.20 \pm 0.03$ & 0.01 \\
\hline e & $56.6^{*}$ & $0.50 \pm 0.01$ & $6.0 \pm 0.6$ & $35 \pm 3$ & $0.20 \pm 0.05$ & 0.01 \\
\hline
\end{tabular}

Table S1: Parameters fitted to Eq. (E7) and obtained in the cases reported in Figs. 1 and 2. In all the cases the dipolar coupling was kept fixed to $b / 2 \pi=-2117.0 \mathrm{~Hz}$. The errors have been estimated by a Monte Carlo approach in which an experimental error of $1 \%$ on the $s(\tau)$ was assumed. * kept fixed. **Root Mean Square Deviation: 
$\operatorname{RMSD}=\left(\Sigma_{\mathrm{i}}\left(\mathrm{y}_{\mathrm{i}}{ }^{\mathrm{exp}}-\mathrm{y}_{\mathrm{i}}^{\mathrm{cal}}\right)^{2} / \mathrm{N}\right)^{1 / 2}$ with $\mathrm{y}_{\mathrm{i}}{ }^{\text {exp }}$ being the experimental points $s(\tau), \mathrm{y}_{\mathrm{i}}^{\text {cal }}$ the predicted ones and $\mathrm{N}$ the number of points.

In order to estimate the error in the determination of the dipolar coupling by this method, Table S2 presents fits of the same spin-echo data, where the dipolar coupling is a free parameter, while the angle deviation $\Delta$ was fixed to the corresponding value in Table 1. Strategies for the independent determination of $\Delta$ are discussed in section S5.

\begin{tabular}{|r|r|r|r|r|r|r|c|}
\hline & $\mathbf{J} / \mathbf{H z}$ & $\mathbf{b}_{\mathbf{j k}} / \mathbf{H z}$ & $\Delta /{ }^{\circ}$ & $\mathbf{T}_{\mathbf{2}}^{\mathbf{0}} / \mathbf{m s}$ & $\mathbf{T}_{\mathbf{2}}^{\mathbf{J}} / \mathbf{m s}$ & $\mathbf{p}$ & $\mathbf{R M S D} * * \mathbf{H z}$ \\
\hline $\mathbf{a}$ & $56.6^{*}$ & $-2125 \pm 3$ & $-0.49^{*}$ & $1.7 \pm 0.1$ & $44.7 \pm 0.1$ & $0.17 \pm 0.01$ & 0.02 \\
\hline $\mathbf{b}$ & $56.6^{*}$ & $-2141 \pm 7$ & $-0.26^{*}$ & $3.3 \pm 0.1$ & $76 \pm 1$ & $0.29 \pm 0.01$ & 0.02 \\
\hline $\mathbf{c}$ & $56.6^{*}$ & $-2136 \pm 7$ & $0.26^{*}$ & $4.6 \pm 0.1$ & $42.7 \pm 0.5$ & $0.20 \pm 0.01$ & 0.01 \\
\hline $\mathbf{d}$ & $56.6^{*}$ & $-2104 \pm 4$ & $0.50^{*}$ & $6.0 \pm 0.1$ & $34.9 \pm 0.1$ & $0.20 \pm 0.01$ & 0.01 \\
\hline
\end{tabular}

Table S2: Parameters fitted to Eq. (E7) and obtained in the cases reported in Fig. 2. In all the cases the angle deviation $\Delta$ was fixed to the value determined in the fitting procedure described by Table 1. The errors have been estimated by a Monte Carlo approach in which an experimental error of $1 \%$ on the $s(\tau)$ was assumed. * kept fixed. **Root Mean Square Deviation: $\operatorname{RMSD}=\left(\Sigma_{\mathrm{i}}\left(\mathrm{y}_{\mathrm{i}}^{\text {exp }}-\mathrm{y}_{\mathrm{i}}^{\text {cal }}\right)^{2} / \mathrm{N}\right)^{1 / 2}$ with $\mathrm{y}_{\mathrm{i}}^{\text {exp }}$ being the experimental points $s(\tau), \mathrm{y}_{\mathrm{i}}^{\mathrm{cal}}$ the predicted ones and $\mathrm{N}$ the number of points.

Figure S2 presents expanded regions of the ${ }^{1} \mathrm{H}$-decoupled ${ }^{13} \mathrm{C}$ NMR spectra of ${ }^{13} \mathrm{C}_{2}$ glycine for the five angle settings at which the spin-echo data presented in Figure 2 of the manuscript was recorded. While the lineshapes for the carbonyl resonance show a noticeable broadening for offsets of $\pm 0.5^{\circ}$ (Figure S2(a) and (e)) as compared to the onangle case (Figure (S2(c)), the width of the lineshapes for the $\mathrm{CH}_{2}$ resonance are very similar. Specifically, the linewidths (as measured at $20 \%$ of the full maximum height, so as to take into account the true width of the carbonyl resonance in Figure $\mathrm{S} 2(\mathrm{e}))$ are $\mathrm{CH}_{2}$ : 
$141 \mathrm{~Hz}$ (on angle, Figure S2(c)), $158 \mathrm{~Hz}\left(\Delta=-0.5^{\circ}\right.$, Figure S2(a)), $151 \mathrm{~Hz}\left(\Delta=+0.5^{\circ}\right.$, Figure S2(e)) and CO: $126 \mathrm{~Hz}$ (on angle, Figure S2(c)), $177 \mathrm{~Hz}\left(\Delta=-0.5^{\circ}\right.$, Figure S2(a)), $182 \mathrm{~Hz}\left(\Delta=+0.5^{\circ}\right.$, Figure S2(e)). This observation is to be expected given the larger CSA for the carbonyl resonance. Note that off-MAS spin-echo curves are obtained for both resonances in a dipolar-coupled pair, i.e., at both the ${ }^{13} \mathrm{C} \mathrm{CO}$ and $\mathrm{CH}_{2}$ resonances. Indeed, the spin-echo curves in Figures 1 and 2 of the main text are for the $\mathrm{CO}$ and $\mathrm{CH}_{2}$ resonances, respectively - a comparison of the fitted data for Figure 1(b) and Figure 2(b) presented in Table S1 shows that the determined offsets are the same within experimental error $\left(\Delta=-0.27 \pm 0.01^{\circ}\right.$ Figure $1(\mathrm{~b})$ and $\Delta=-0.26 \pm 0.01^{\circ}$ Figure 2(b)). Considering future applications to real systems, it can be concluded that even in unfavourable cases where the broadening of the carbonyl resonance may be problematic, spin-echo modulation curves with the inherent dipolar coupling information can still be obtained for aliphatic resonances. 


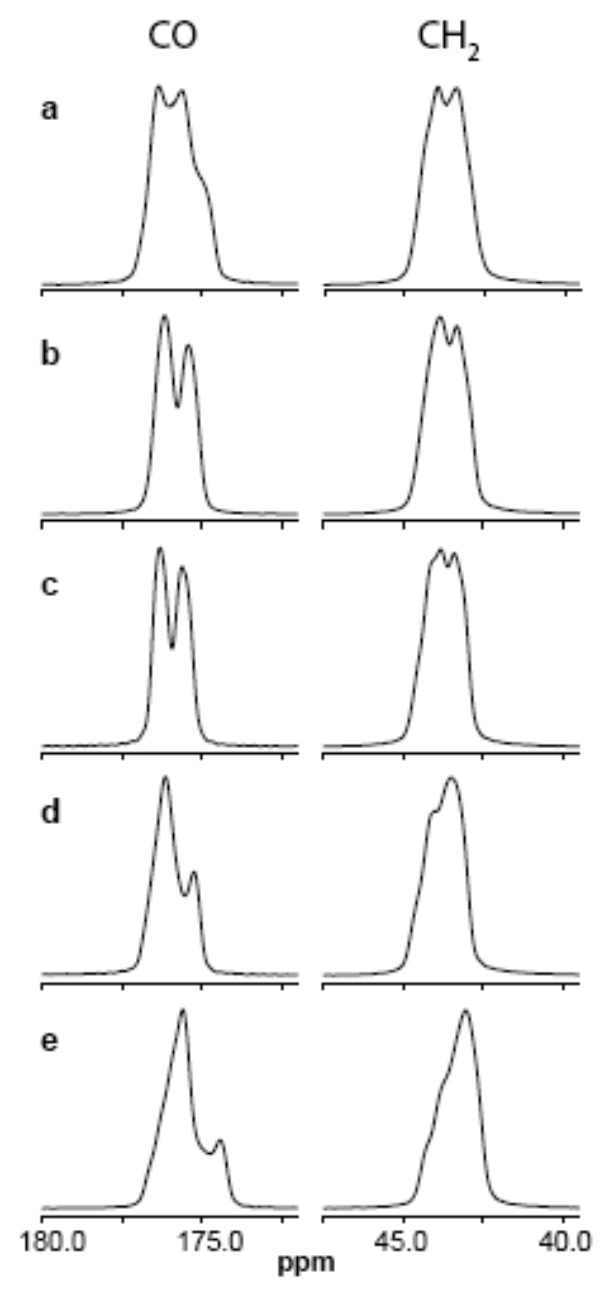

Figure S2: Expanded regions of ${ }^{1} \mathrm{H}$-decoupled ${ }^{13} \mathrm{C}$ NMR spectra of ${ }^{13} \mathrm{C}_{2}$ glycine for the five angle settings at which the spin-echo data presented in Figure 2 of the manuscript was recorded. The spectra in (b) and (c) are also presented in Figure 1 of the manuscript.

\section{(S5) Spinning Angle Determination}

The purpose of our communication is to demonstrate the phenomenon whereby residual dipolar couplings are introduced in spin-echo MAS experiments recorded at rotor angles corresponding to small deviations from the magic angle. In this section, we address the question as to how the spinning angle can be accurately determined, with this being a prerequisite for the practical use of the phenomenon to quantitatively determine 
unknown dipolar couplings. The measurement of an unknown distance is beyond the scope of this initial communication.

Our suggested approach for determining the angle is to measure spin-echo curves for rotors packed separately with a calibration sample and the sample for which an unknown distance is to be characterised. A suitable calibration sample for the determination of unknown ${ }^{13} \mathrm{C}-13 \mathrm{C}$ dipolar couplings is the model compound ${ }^{13} \mathrm{C}_{2}$ glycine; as shown above (see Table S1), fits to spin-echo data allow the determination of the spinning angle to an accuracy of $\pm 0.01^{\circ}$. This method relies on the angle setting being unperturbed upon ejecting and inserting a rotor. In this respect, we note that this approach is relied upon in ST MAS experiments: the obtaining of high-resolution spectra in such ST MAS experiments requires the magic angle to be set very accurately (better than $0.002^{\circ}$ ). When applying the ST MAS experiment to samples giving low sensitivity, it is usual practice to calibrate the angle using a standard and then switch rotor to study the sample of interest. ${ }^{9}$ When using the off-angle spin-echo phenomenon described here, a best practice protocol would involve measuring a spin-echo curve (each curve in Figure 2 of the manuscript took only 11 minutes to record) for the calibration sample both before and after the measurement for the sample being characterised.

The Southampton group has recently described the development of a mechanism based on the Hall effect for the accurate electronic monitoring of the angle setting, independent of the NMR experiment; initial results from the prototype design show that the angle setting is determined to an accuracy of at least $\pm 0.02^{\circ} .{ }^{10}$ The use of such technology would avoid the need for using a calibration sample as described above.

As an independent check of the validity of the fitting of the spin-echo data using the theoretical function (see Eqs. (E10 to E12) in section S3), for each angle setting, a separate ${ }^{13} \mathrm{C}$ CP MAS spectrum was recorded for a sample of unlabelled $L$-alanine (packed in a different rotor to that containing the labelled glycine sample). The spinning angle was determined by the best fit (by minimising the root mean square deviation between the experimental and the simulated data) for the $\mathrm{CO}_{2}{ }^{-}$resonance (see Figure S3) to spectra simulated using SIMPSON ${ }^{11}$ assuming CSA parameters of $\delta=-70 \mathrm{ppm}$ and $\eta$ $=0.78,{ }^{12}$ with $7 \mathrm{~Hz}$ of exponential line broadening applied to the simulated free-induction decay. While it is reassuring that the angle settings determined by this procedure (see 
Table S3) are in agreement with those determined by fits of the spin-echo data to Eq. (E7) in Table S1, the accuracy of this method is estimated to be at best $\pm 0.05^{\circ}$. (A significant source of systematic error is due to the degree of applied line broadening affecting the fit). We thus recommend the alternative angle calibration protocol described above. (Note that we have verified that the lineshape for the $\mathrm{CO}_{2}{ }^{-}$resonance in a ${ }^{13} \mathrm{C}$ spectrum for an off-angle setting is unchanged upon ejecting and reinserting the rotor.)

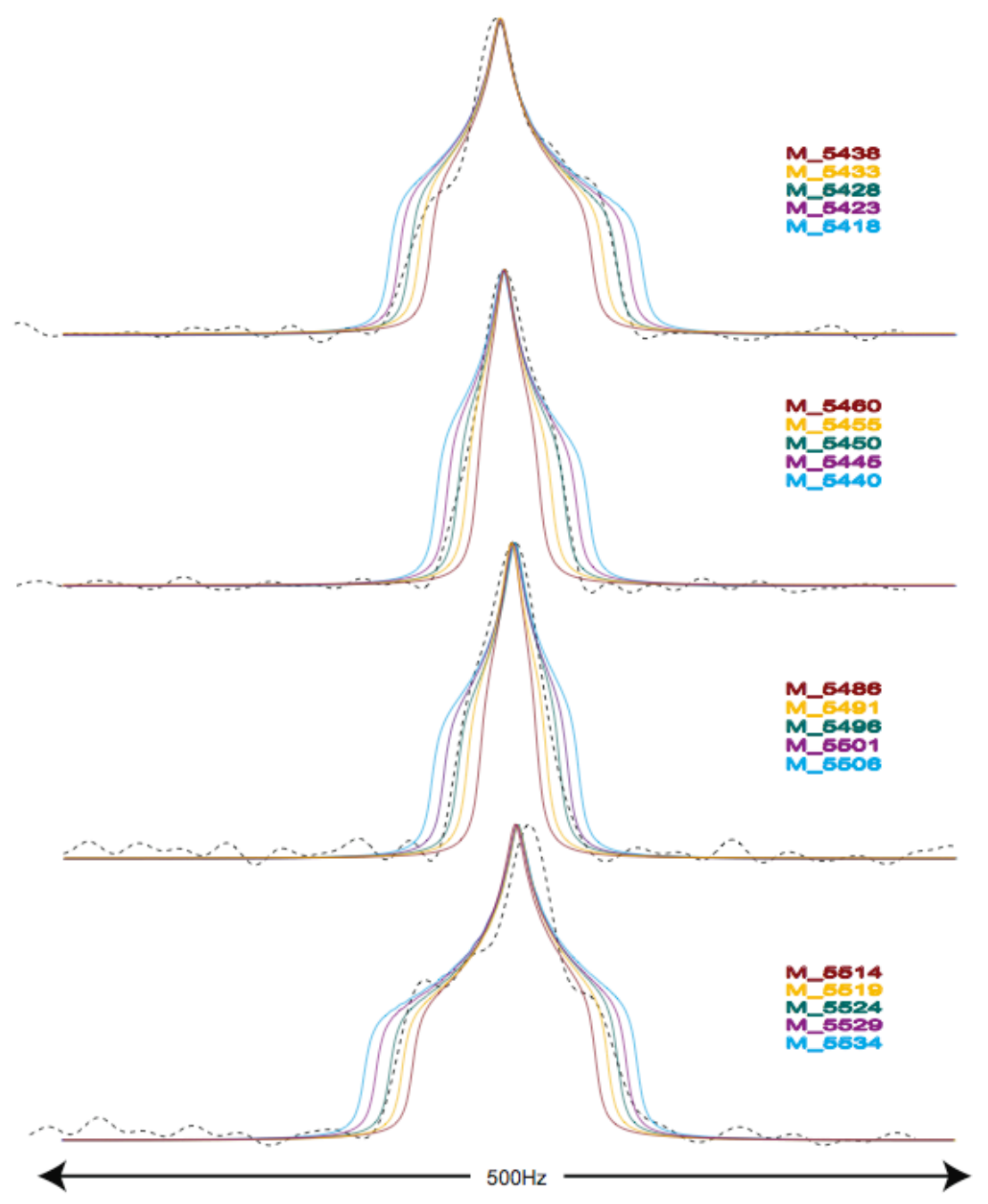

Figure S3 Best fits (see Table S3) for the $\mathrm{CO}_{2}{ }^{-}$resonance in ${ }^{13} \mathrm{C} \mathrm{CP}$ sample-spinning experiments of $L$ alanine at natural abundance, corresponding to the angle settings used for the off-angle spin-echo experiments on ${ }^{13} \mathrm{C}_{2}$ glycine in Figure 2 of the main text. The experimental spectra are shown as dashed lines. Spectra were simulated using SIMPSON $^{11}$ assuming CSA parameters of $\delta=-70 \mathrm{ppm}$ and $\eta=0.78,{ }^{12}$ with $7 \mathrm{~Hz}$ of 
exponential line broadening applied to the simulated free-induction decay. In addition to the best-fit angle settings (from top to bottom: $\Delta=-0.46^{\circ},-0.24^{\circ},+0.22^{\circ},+0.48^{\circ}$ ), spectra are simulated for angle settings $\pm 0.05^{\circ}$ and $\pm 0.1^{\circ}$ away.

\begin{tabular}{|c|c|c|c|}
\hline & $\begin{array}{l}\Delta /{ }^{\circ} \text { (alanine } \\
\text { lineshape) }\end{array}$ & RMSD* & $\Delta /{ }^{\circ}$ (Table S1) \\
\hline $\mathrm{a}$ & $-0.46 \pm 0.05$ & 0.028 & $-0.49 \pm 0.01$ \\
\hline $\mathrm{b}$ & $-0.24 \pm 0.05$ & 0.017 & $-0.26 \pm 0.01$ \\
\hline $\mathrm{d}$ & $+0.22 \pm 0.05$ & 0.024 & $+0.26 \pm 0.01$ \\
\hline $\mathrm{e}$ & $+0.48 \pm 0.05$ & 0.030 & $+0.50 \pm 0.01$ \\
\hline
\end{tabular}

Table S3: A comparison of the determination of the deviation $\Delta$ (in degrees) from the magic angle using the fitting of the alanine $\mathrm{CO}_{2}{ }^{-}$resonance (see Figure S2) and the fitting of spin-echo data to Eq. (E7) for the data presented in Figure 2 of the manuscript (see Table 1 in section S4). *Root Mean Square Deviation: $\operatorname{RMSD}=\left(\Sigma_{\mathrm{i}}\left(\mathrm{y}_{\mathrm{i}}{ }^{\mathrm{exp}}-\mathrm{y}_{\mathrm{i}}{ }^{\mathrm{cal}}\right)^{2} / \mathrm{N}\right)^{1 / 2}$ with $\mathrm{y}_{\mathrm{i}}{ }^{\text {exp }}$ being the experimental points $s(\tau), \mathrm{y}_{\mathrm{i}}^{\text {cal }}$ the predicted ones and $\mathrm{N}$ the number of points.

\section{(S6) Applicability of the Phenomenon for Measuring Smaller Dipolar Couplings}

For the case of a one-bond ${ }^{13} \mathrm{C}-13 \mathrm{C}$ dipolar coupling in glycine, this work has clearly demonstrated that the introduction of residual dipolar couplings leads to marked perturbations in the observed spin-echo evolution for small deviations $\left(\Delta<0.5^{\circ}\right)$ from the magic angle. While there is importance in the accurate measurement of one-bond distances, e.g., Ref. ${ }^{13}$ uses this approach to probe protein-induced bonding perturbations of the rhodopsin chromophore, this section addresses the important issue of the applicability of the phenomenon to measuring smaller dipolar couplings. Of particular interest are ${ }^{13} \mathrm{C}-{ }^{13} \mathrm{C}$ distances in the range $4-5 \AA$ that provide important constraints in characterising biomolecular structures. Dipolar couplings corresponding to such distances can be determined using symmetry based recoupling techniques ${ }^{14}$ or the rotational 
resonance phenomenon, ${ }^{15}$ an example being the characterisation of the structure of the transmembrane dimer interface of glycophorin A in membrane bilayers. ${ }^{16}$

Figure S4 presents plots of Eq. (E7) for the case of a dipolar coupling constant, $b / 2 \pi$ equal to (a) $-120 \mathrm{~Hz}$ and (b) $-60 \mathrm{~Hz}$, corresponding to ${ }^{13} \mathrm{C}-{ }^{13} \mathrm{C}$ distances of 4 and $5 \AA$, respectively. Since such a distance usually corresponds to a through-space proximity rather than a through-bond connectivity, the $J$ coupling is set to zero. For a ${ }^{13} \mathrm{C}-{ }^{13} \mathrm{C}$ distance of $4 \AA$ and an offset of $\Delta=2^{\circ}$ from the magic angle, the first zero crossing is observed at a spin-echo duration, $\tau$, equal to $\sim 125 \mathrm{~ms}$ (see Figure $\mathrm{S} 4(\mathrm{a})$ ), while for a ${ }^{13} \mathrm{C}$ ${ }^{13} \mathrm{C}$ distance of $5 \AA$, an offset of $\Delta=3^{\circ}$ from the magic angle corresponds to a first zero crossing at a spin-echo duration, $\tau$, equal to $\sim 150 \mathrm{~ms}$ (see Figure S4(b)). Thus, if it is possible to record such spin-echo experiments out to a spin-echo duration, $\tau$, of $\sim 200 \mathrm{~ms}$, it should be feasible to measure ${ }^{13} \mathrm{C}-13 \mathrm{C}$ distances in the range $4-5 \AA$ at offsets of $\Delta=2-$ $3^{\circ}$ from the magic angle.

For conventional high-power ${ }^{1} \mathrm{H}$ decoupling, it is not recommended to exceed decoupling times of $50 \mathrm{~ms}$. Longer spin-echo durations up to and beyond $200 \mathrm{~ms}$ are, however, possible if alternative low-load ${ }^{1} \mathrm{H}$ decoupling approaches are employed. Specifically, it has been recently shown that a ${ }^{15} \mathrm{~N}-15 \mathrm{~N} J$ coupling of $3.8 \mathrm{~Hz}$ can be determined in a spin-echo (on-angle) MAS $\left(v_{\mathrm{R}}=22.5 \mathrm{kHz}\right)$ experiment whereby a maximum spin-echo duration, $\tau$, of $200 \mathrm{~ms}$ was used, such that the first zero crossing is observed at a spin-echo duration, $\tau$, of $130 \mathrm{~ms} .{ }^{17}$ In these experiments, rotor-synchronised Hahn-echo pulse train (RS-HEPT) ${ }^{18}{ }^{1} \mathrm{H}$ decoupling with a duty cycle of only $6 \%$ was used. In further work considering spin-echo experiments recorded at $30 \mathrm{kHz}$ MAS using ${ }^{13} \mathrm{CH}$-labelled alanine, it has been found that the dephasing time, $T_{2}{ }^{\prime}$, using the same RSHEPT approach is about two thirds of that achievable with (experimentally-optimised) 4 TPPM. Moreover, there is a marked improvement upon increasing MAS frequency, and 10 -spin simulations carried out using SPINEVOLUTION ${ }^{19}$ reveal a doubling of $T_{2}{ }^{\prime}$ at 60 $\mathrm{kHz}$ MAS as compared to at $30 \mathrm{kHz}$ MAS. ${ }^{20,21}$ Indeed, we envisage that such very-fast MAS frequencies that are now experimentally achievable (the application to a protein sample was described recently by Ernst et al. ${ }^{22}$ ) will be very important in the context of spin-echo experiments and in particular the off MAS phenomenon. In this context, we 
also note the interesting phenomenon that there is an evident increase in the fitted dephasing times, $T_{2} \mathrm{~J}$, for off magic-angle spin-echo experiments (see Table S1). While the origin of this phenomenon is currently being investigated in our groups, we emphasise the benefit this brings in allowing the effects of residual dipolar couplings to be evident in off-angle spin-echo modulation curves.

To further support our arguments concerning the feasibility of using the off-MAS phenomenon to measure longer $\mathrm{C}$ - $\mathrm{C}$ distances, we note that it is possible to record independent spin-echo data sets at different angle offsets. Since the to-be-fitted dipolar coupling constant is the same for all such data sets, an approach involving the fitting of such an ensemble of spin-echo data sets will improve the error associated with the determination of the dipolar coupling constant. In this way, the dipolar coupling constant can be more accurately determined for shorter spin-echo durations as would be the case for a single spin-echo data set.

Angle offsets of several degrees do lead to appreciable broadening of the NMR signals. However, for aliphatic ${ }^{13} \mathrm{C}$ resonances with small CSAs, the broadening at such angle offsets is not extreme; as an example, Figure S5 presents a comparison of the $\mathrm{CH}_{3}$, $\mathrm{CH}$ and $\mathrm{COO}^{-}$resonances for ${ }^{13} \mathrm{C}$ spectra of $\mathrm{U}^{-13} \mathrm{C} \mathrm{L}$-alanine recorded at the magic angle and an offset of $-3.3^{\circ}$ (as determined by a fit of the $\mathrm{COO}^{-}$lineshape). The $\mathrm{CH}$ and $\mathrm{CH}_{3}$ resonances are broadened by a factor of 3-4 in the off-angle spectrum. This limited degree of broadening will be acceptable in many applications. In this context, we reiterate the arguments made in the concluding paragraph of the main text: One of the strengths of the solution-state and solid-state RDC approaches is that the dipolar coupling information is introduced without breaking the weak-coupling approximation. This makes it possible to use frequency-selective radiofrequency pulses to isolate individual couplings from a multiple-spin coupled network. The simple effect described here, thus, holds out the promise of the robust estimation of selected spin-spin couplings in multiple-spin systems.

Furthermore, we are currently developing technology in which small, fast, and precise changes in the spinning angle are achieved during the NMR pulse sequence. Such methods should make it possible to estimate long-range distances without loss of spectral resolution, even in multiply-labelled samples. 
Finally, it is to be emphasised that the phenomenon can also be applied to the determination of other homonuclear dipolar couplings, an interesting area for potential development being ${ }^{19} \mathrm{~F}-19 \mathrm{~F}$ dipolar couplings. ${ }^{23}$

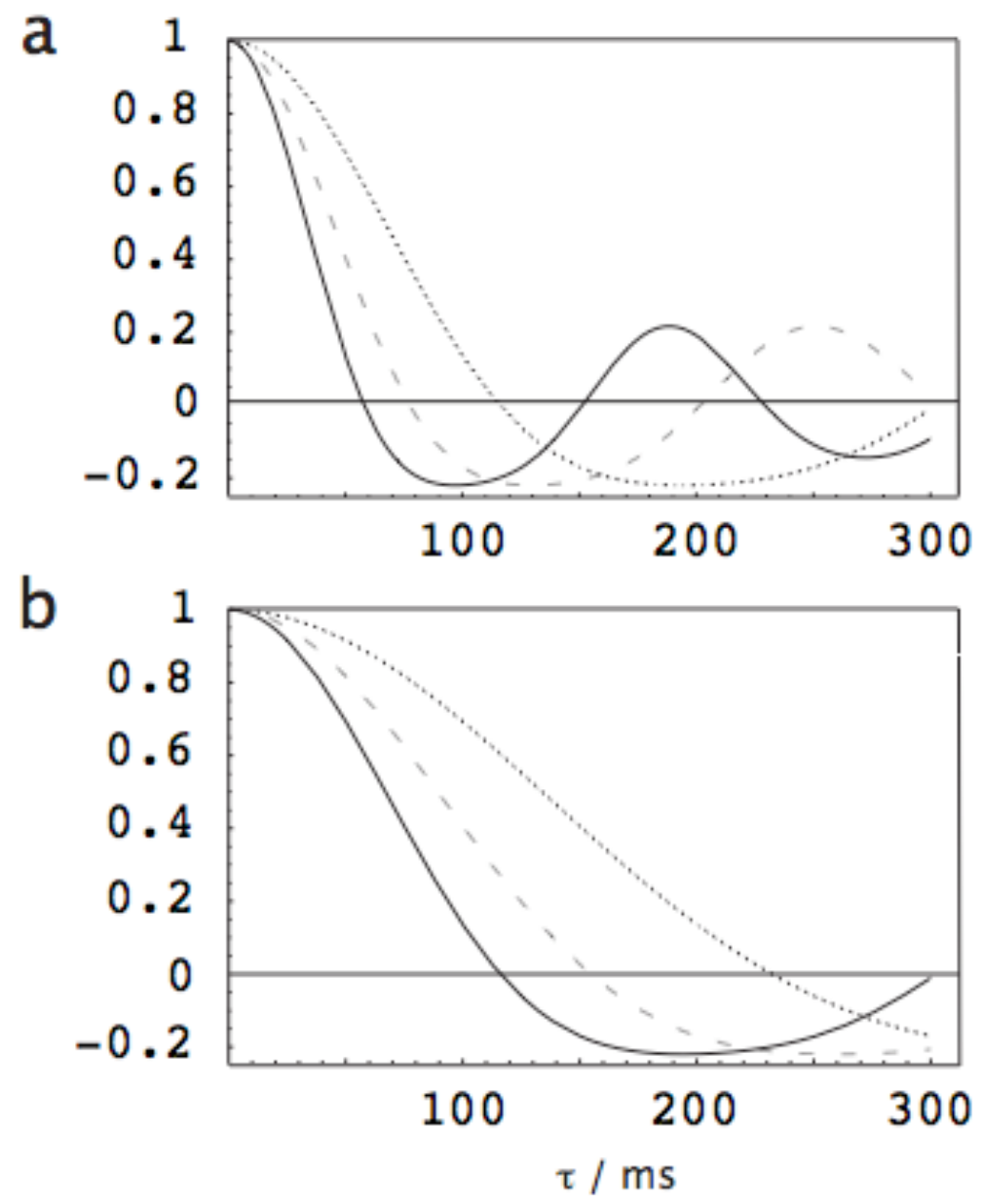

Figure S4: Plots of Eq. (E7) for the case of a dipolar coupling constant, $b / 2 \pi$ equal to (a) $-120 \mathrm{~Hz}$ and (b) $-60 \mathrm{~Hz}$, corresponding to ${ }^{13} \mathrm{C}-13 \mathrm{C}$ distances of 4 and $5 \AA$, respectively. Dotted, dashed and normal lines correspond to angle offsets of $\Delta=2,3,4^{\circ}$, respectively. $J$ and $p$ are set equal to zero. No exponential damping is applied (corresponding to a infinitely long $T_{2}^{J}$ value). 

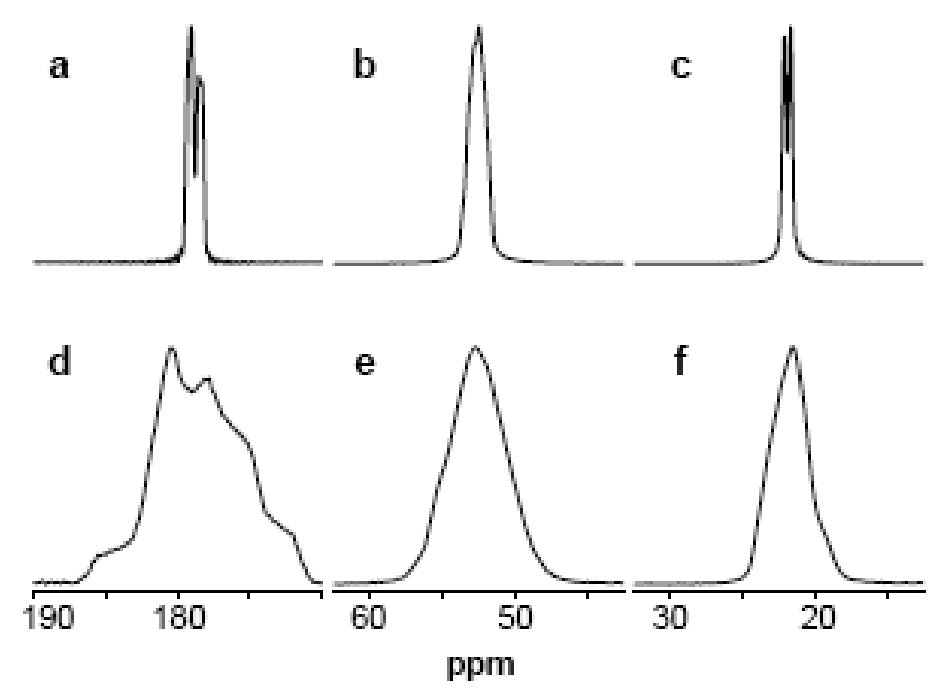

Figure S5: A comparison of the (a, d) $\mathrm{COO}^{-}$, (b, e) $\mathrm{CH},(\mathrm{c}, \mathrm{f}) \mathrm{CH}_{3}$ resonances for ${ }^{13} \mathrm{C}$ spectra $(7.1 \mathrm{~T})$ of $\mathrm{U}-{ }^{13} \mathrm{C} L$-alanine recorded at $(\mathrm{a}, \mathrm{b}, \mathrm{c})$ the magic angle and $(\mathrm{d}, \mathrm{e}, \mathrm{f})$ an offset of $-3.3^{\circ}$ (as determined by a fit of the $\mathrm{COO}^{-}$lineshape).

\section{(S7) References}

(1) Hediger, S.; Meier, B. H.; Kurur, N. D.; Bodenhausen, G.; Ernst, R. R. Chem. Phys. Lett. 1994, 223, 283.

(2) Metz, G.; Wu, X. L.; Smith, S. O. J. Magn. Reson. Ser. A 1994, 110, 219.

(3) Bennett, A. E.; Rienstra, C. M.; Auger, M.; Lakshmi, K. V.; Griffin, R. G. J. Chem. Phys. 1995, 103, 6951.

(4) De Paepe, G.; Giraud, N.; Lesage, A.; Hodgkinson, P.; Bockmann, A.; Emsley, L. J. Am. Chem. Soc. 2003, 125, 13938.

(5) De Paepe, G.; Hodgkinson, P.; Emsley, L. Chem. Phys. Lett. 2003, 376, 259.

(6) Potrzebowski, M. J.; Tekely, P.; Dusausoy, Y. Solid State Nucl. Magn. Reson. 1998, 11, 253.

(7) Duma, L.; Lai, W. C.; Carravetta, M.; Emsley, L.; Brown, S. P.; Levitt, M. H. ChemPhysChem 2004, 5, 815.

(8) Pileio, G.; Concistrè, M.; McLean, A.; Gansmuller, A.; Brown, R. C. D.; Levitt, M. H. J. Magn. Reson., in press.

(9) Ashbrook, S. E.; Wimperis, S. Prog. Nucl. Magn. Reson. Spectrosc. 2004, 45, 53.

(10) Mamone, S.; Dorsch, A.; Johannessen, O. G.; Naik, M. V.; Madhu, P. K.; Levitt, M. H., J. Magn. Reson. accepted.

(11) Bak, M.; Rasmussen, J. T.; Nielsen, N. C. J. Magn. Reson. 2000, 147, 296.

(12) Levitt, M. H.; Eden, M. Mol. Phys. 1998, 95, 879. 
(13) Carravetta, M.; Zhao, X.; Johannessen, O. G.; Lai, W. C.; Verhoeven, M. A.; Bovee-Geurts, P. H. M.; Verdegem, P. J. E.; Kiihne, S.; Luthman, H.; de Groot, H. J. M.; deGrip, W. J.; Lugtenburg, J.; Levitt, M. H. J. Am. Chem. Soc. 2004, $126,3948$.

(14) Carravetta, M.; Eden, M.; Johannessen, O. G.; Luthman, H.; Verdegem, P. J. E.; Lugtenburg, J.; Sebald, A.; Levitt, M. H. J. Am. Chem. Soc. 2001, 123, 10628.

(15) Dusold, S.; Sebald, A. In Annual Reports On NMR Spectroscopy; Academic Press Inc: San Diego, 2000; Vol. 41, pp 185.

(16) Smith, S. O.; Song, D.; Shekar, S.; Groesbeek, M.; Ziliox, M.; Aimoto, S. Biochemistry 2001, 40, 6553.

(17) Pham, T. N.; Griffin, J. M.; Masiero, S.; Lena, S.; Gottarelli, G.; Hodgkinson, P.; Filip, C.; Brown, S. P. Phys. Chem. Chem. Phys. 2007, 9, 3416.

(18) Filip, X.; Tripon, C.; Filip, C. J. Magn. Reson. 2005, 176, 239.

(19) Veshtort, M.; Griffin, R. G. J. Magn. Reson. 2006, 178, 248.

(20) Brown, S. P. In EUROMAR: Tarragona, Spain, 2007.

(21) Griffin, J. M.; Tripon, C.; Filip, C.; Brown, S. P., in preparation.

(22) Ernst, M.; Meier, M. A.; Tuherm, T.; Samoson, A.; Meier, B. H. J. Am. Chem. Soc. 2004, 126, 4764.

(23) Ulrich, A. S. Prog. Nucl. Magn. Reson. Spectrosc. 2005, 46, 1. 